This is the peer reviewed version of the following article: Hood, Rick, Gorin, Sarah, Goldacre, Allie, Muleya, Wilson and Bywaters, Paul (2020) Exploring drivers of demand for child protection services in an English local authority. Child \& Family Social Work, 25(3), pp. 657-664., which has been published in final form at https:// doi.org/10.1111/cfs.12740. This article may be used for non-commercial purposes in accordance with Wiley Terms and Conditions for Use of Self-Archived Versions. 


\section{Exploring drivers of demand for child protection services in an English local authority}

\section{Abstract}

This paper reports on an empirical study of child protection services in a local authority where rates of investigations and interventions rose to unprecedented levels during the course of a single year. The aim of the research was to explore explanations for this rise in demand among the providers of children's social care in the area. Using an interpretative qualitative design, a series of focus groups and interviews were carried out with practitioners and managers $(n=25)$ from statutory services and Early Help. The findings identified a combination of long-term and short-term drivers of demand. Long-term factors emphasised the impact of rising levels of deprivation combined with cuts to community-based services for children and young people. Short-term factors ranged from a more proactive approach to child neglect to more effective multi-agency partnerships and joint decisionmaking. The interaction between these factors was found to be accentuating an underlying shift to 'late intervention' across the sector. The findings are contextualised in relation to contemporary debates about the crisis of demand for children's social care and the complex relationship between prevention and protection. 


\section{Introduction}

Recent years have seen increasing concerns about spiralling demand for children's social care (CSC) in England (Action for Children et al., 2017; ADCS, 2018; National Audit Office, 2019). Here CSC is taken to mean statutory provision for children in need (CIN) and children at risk of neglect and abuse, as well as services for children in public care. The rising cost of CSC - particularly for child protection (CP) and care - is placing huge financial pressure on the local authorities (LAs) that provide these services (e.g. Butler, 2018). The usual explanation boils down to a combination of 'poverty plus cuts', beginning with the 2008 financial crisis. The latter precipitated an extended period of austerity and 'state shrinkage' that is still being pursued by the (UK) central government. Austerity policies have disproportionately affected deprived areas (mostly outside the wealthy Southeast region centred on London), which came to rely on public sector investment in the decades following de-industralisation in the 1980s. Mismanagement of social policy during this period, particularly in relation to housing and benefits payments (O'Hara, 2015), has compounded the hardship faced by families and exacerbated the risk factors associated with child abuse and neglect (Bywaters et al., 2016). Meanwhile, budgetary pressures have forced LAs to cut universal and preventive services in order to protect core statutory provision. The removal of community-based support means that problems are more likely to escalate to the point where statutory intervention becomes necessary. Across the country, CSC has undergone a shift to 'late intervention', with spending on child protection and children in public care increasing even as other forms of provision have been stripped back. LAs risk a vicious circle of counterproductive rationing, as they struggle to avoid overspending and (for some) de-facto bankruptcy.

The 'poverty plus cuts' narrative has its antecedents in a longstanding debate about the balance between prevention and protection in child welfare services (e.g. Department of Health, 1995). The contemporary picture contrasts a sustained rise in demand for $\mathrm{CP}$ and care with fluctuating (but not greatly increasing) levels of referrals and CIN over the past eight years (Hood et al., 2019; National Audit Office, 2019). While deprivation is generally accepted as a key driver of demand, its effects on provision are less well understood. Hood et al.'s (2019) analysis of government figures showed that LAs with high levels of deprivation (and therefore demand) are likely to screen out more cases, particularly in the initial stages of referral and assessment, and to close CIN cases earlier. This screening mechanism may help explain why rates of CIN have been more stable relative to $\mathrm{CP}$ (although it would not explain why referrals have not risen). It also indicates that as services become increasingly stretched the tendency to gate-keep and ration services will increase the proportion of work geared around core statutory duties. It has also been suggested that institutional anxiety 
about Ofsted inspections may be encouraging some LAs to do more CP relative to referrals; some evidence of this was found by Hood et al. (2016) in connection with LAs receiving an inadequate rating although their results have yet to be replicated.

Other explanations for the crisis also focus on provision and the way services respond to demand. One approach is to study variations between LAs, including those with similar levels of expenditure or deprivation, in order to explore the importance of LA characteristics such as 'custom and practice' (National Audit Office, 2019). Another is to study individual and regional differences in the chances of receiving a $\mathrm{CP}$ intervention, which point to inequalities in the way services are provided (Bywaters, 2017). Ofsted, the CSC inspectorate, has argued that quality of leadership is a key factor in the best performing LAs (Ofsted, 2015) while, somewhat counter-intuitively, workforce stability (as measured by turnover) seems to be associated with higher rates of children in care (Wijedasa et al., 2018). Another approach is to study flaws in the system that might be hindering services from working effectively with families (Featherstone et al., 2018). Such flaws include the stigmatisation of poverty, excessive managerialism, blame culture and defensive practice, a preoccupation with risk, a tendency to focus on parenting deficits, and a rigid and bureaucratic culture of provision (Featherstone et al., 2018). Whichever explanation is preferred, there is clearly a need to understand better the factors driving demand, some of which will reflect national trends and others the varying characteristics of regions and local areas. In what follows, the paper outlines a conceptual framework for understanding demand before reporting on an empirical study in an English local authority.

\section{Conceptualising demand for CSC}

Although widely used, demand is a rather ambiguous and problematic term in CSC. As with any publicly provided welfare service, the economic theory of demand lies in tension with the psychosocial theory (or theories) of need. A shared assumption is that people make choices in order to satisfy their goals and aspirations, and in doing so must negotiate for resources and support from others. In economic terms, demand is in an equilibrating relationship with supply ('provision'), which is primarily governed by the price mechanism in competitive markets (Lewis \& Widerquist, 2001). Neo-liberal reforms to the welfare state have therefore tried to create effective and efficient markets of care for the citizen-consumer. In psychosocial terms, need is experienced by the individual but also produced and mediated by the social environment (Maslow, 1970). By extension, need may be determined by laws and social norms, or categorised by institutions and professionals, 
in ways that differ from individual experience (Bradshaw, 1972). Such distinctions relate only approximately to the economic concepts of demand and supply. What is more, any price mechanism for allocating resources to needs is complicated by the fact that most CSC services are funded by the state (and ultimately taxpayers) rather than the individuals receiving them. Among other things, this means that while there may be many providers of child welfare services, there is effectively only one buyer (i.e. the state) - a market situation known as 'monopsony' (Manning, 2003). This also means that utilitarian rationing processes will constrain the degree to which supply is able to increase to meet demand, obliging agencies to use professional assessments and eligibility criteria to determine who receives what kind of service.

Another peculiarity of demand for CSC is the distinction between services that are requested (or at least voluntarily received) by service users and services that are provided irrespective of consent in order to safeguard children's welfare. The former can be more readily equated with the provision of services in commercial markets. The latter is effectively a form of social regulation (Sparrow, 2000), which is not usually welcomed by its direct recipients but is mandated by law (and by extension the general public) in order to reduce social harms. Identifying and preventing child abuse, which mostly occurs within private family life and sometimes involves deliberate avoidance and deception on the part of the abuser, requires regulatory activities such as surveillance, investigation and intervention - again not usually a feature of everyday service provision. In social work practice these distinctions are often represented as dichotomous: care vs control, prevention vs protection, consent vs coercion, etc. (Howe, 2014). In organisational terms, agencies are often expected to discharge their regulatory role within a broader remit of service provision, so that the distinction between these types of work may be insufficiently recognised (hence the awkwardness of referring to 'demand' for child protection investigations and care proceedings). Moreover, the trade-off between regulatory objectives and the expectations of service providers means that accountability pressures may push agencies to prioritise one at the expense of the other, while only recognising performance in the category that is being prioritised (Hood, 2019).

Even from this brief overview it will be apparent that demand for CSC is heterogenous and imperfectly matched to provision. It includes elements of need that may be articulated by service users but not met by providers, e.g. due to constraints around resources or remit, as well as needs (or risks) that may not be articulated by users but require regulatory intervention. It is this multilayered concept of demand that was investigated in the study described below. 


\section{Methods}

The aim of the study was to explore the reasons for a rise in demand for child protection in 'Homeshire', a single English LA in the south of England. Homeshire is in the upper tercile of deprivation rankings based on the weighted average score of the Index of Multiple Deprivation (IMD), i.e. it is one of the least deprived LAs in the country. Most of the population lives in urban areas defined as city, town or minor conurbation. Its inspection ratings from Ofsted over the past decade have been either 'good' or 'adequate/requires improvement'. As is typical for LAs with low average deprivation, Homeshire also has relatively low levels of demand for CSC compared to the national average, including for CP services. However, from April 2017 to March 2018, the LA saw an unusual spike in demand. Rates of child abuse ('Section 47') investigations and child protection plans increased by over $70 \%$ compared to the previous year, while referrals went up by nearly $50 \%$ and children in need (all children receiving a statutory service) by $60 \%$. Over the year all these indicators apart from referrals reached their highest rate since 2009, the earliest year for which comparable data was available. The spike in CP interventions put CSC services under great pressure, especially since they were resourced to deal with a much lower level of demand. The work reported on below was carried out by the research team over the summer of 2018.

The aim of the study was to identify and explore hypotheses for the main drivers of demand held by local authority managers and practitioners, including the non-statutory 'Early Help' service. The study employed an interpretative qualitative design to elicit explanations of rising demand for CP in Homeshire, drawing on the experience and knowledge of a sample of insider experts. Ethical approval for the study was obtained from the faculty research ethics committee of the principal investigator's institution.

As a piloting exercise, a workshop was held at an away day attended by all managers and practitioners in statutory CSC. The workshop, a plenary session attended by all staff, helped to identify the key areas for exploration in focus groups and interviews. Qualitative data was then collected from a purposive sample of 25 participants from statutory CSC and Early Help Services (see Table 1), who responded to an email invitation to all staff to attend three 90 minute focus groups. Four 45 minute telephone interviews were also held with people who were unable to attend a focus group but wanted to participate in the study. Each focus group (FG) concentrated on a different topic identified during the away day: adolescents (FG1), leadership and management (FG2) and early help (FG3). Scope was allowed for participants to discuss other issues of interest. Interview questions followed the schedule of the focus group (available as an online appendix) that the 
participant would have attended. Focus groups and interviews were audio-recorded with the consent of participants.

Table 1. Sample of participants in focus groups and interviews

Transcripts from interviews and focus groups were imported into qualitative data analysis software (NVivo11). Analysis followed the six-stage procedure of thematic analysis as outlined by Braun et al. (2019). In the first stage, the researchers familiarised themselves with the data by reading and rereading the transcripts, making initial notes on potential codes, and meeting to discuss commonalities and differences in perspective. At this stage it was important to reflect on the assumptions each member of the research team was bringing to the topic, such as prior experience of working in CP practice or doing research on vulnerable adolescents. In the second stage, one member of the research team (SG) carried out a systematic coding of the transcripts based on these initial discussions. Coding focused on the thematic content of the discussion; for example, a discussion in one of the focus groups about the impact of recession on deprivation levels in the community (FG1) was coded under the theme of 'economic hardship'. Another member of the research team $(\mathrm{RH})$ independently reviewed the codes in terms of applicability and consistency across the transcripts. In the third stage, the researchers searched for themes within the initial codes. For example, references to the impact of 'economic hardship' were included within a broader category of 'long-term drivers of demand', which tended to affect levels of need in the community. On the other hand, the contribution of Signs of Safety, a solution-focused approach to child protection casework (Turnell, 2017), was categorised under 'short term drivers', which tended to affect how need was interpreted and treated by child welfare agencies. In the fourth and fifth stages, the thematic categories were reviewed in a series of research meetings and key definitions were agreed. In the sixth stage, the findings were presented to the LA in a confidential report and discussed in a meeting with the senior leadership team. Feedback on the report was included as supplementary data in the form of researcher notes.

\section{Findings}

When asked to explain rising demand for child protection services in their area, participants identified a combination of long and short-term factors. Key long-term factors were cuts to universal and preventative services, economic and financial hardship, demographics and housing. Key short term factors included better partnership working, integration of Early Help, introduction of Signs of Safety, more assertive leadership, and the impact of inspections. 
Participants reported that since 2010 the LA had been forced to make cuts to preventative services such as youth centres, youth outreach and community work, general family support services and family centres. Such services were able to develop relationships with a range of children and families in local communities, offer safe havens to young people experiencing problems at home, and act on safeguarding concerns before they reached the threshold for statutory intervention. Some participants felt that a lack of this kind of provision, particularly for young people, had led to more referrals to CSC since problems were being overlooked until they grew more serious. It also meant a lack of options for follow-up and support to families following statutory intervention. Other participants observed that community-based workers had accumulated a great deal of local knowledge relevant to child safeguarding, such as the availability and selling of drugs, the experience of domestic abuse and involvement in criminal activity. Loss of this background knowledge, either through staff attrition or redeployment into targeted casework, made it harder for services to identify emerging risks to children in the community or indeed the protective elements of community life:

'So you'd know how they lived, you'd know that their kids are cared for even though they are down the road with just their nappy and t-shirt on. You'd know there's a parent at that house watching them and a parent at that house watching this time, you knew how they worked and how they were a community.' [P23]

Prior to the cuts, community-based practitioners had close working relationships with schools, e.g. providing individual support or more general preventative work about healthy relationships and sexual health. One participant considered that youth centres had helped to break down barriers between young people and the police, allowing police officers to visit and meet with young people on an informal basis in order to build relationships and local knowledge. These links were now felt to be more tenuous, widening the gap between communities and statutory services and making it harder for young people to seek professional help from a trusted source:

"So what we can offer them is someone who has a specialism in youth work or somebody to do six sessions... We are talking about quite entrenched behaviours, or we are talking about exploitation ... so what change are we actually expecting to make in those six sessions compared to someone who builds that relationship time and time again and persists and re-visits and becomes that person that young person can trust and turn to? We just don't have it." [P11] 
Many participants recognised the effects of increased economic hardship on families over the past eight years. Some reported visiting more families who were experiencing poverty or acute financial difficulties as a result of cuts in benefits and entitlements, time lapses before new benefits were received, and loss of employment. Increased levels of stress due to economic hardship was thought to be leading to higher levels of conflict within families, parental acrimony and marital breakdown, making it harder for parents to meet their children's needs and pushing up demand for child welfare services.

Housing issues were thought to have contributed to demand for CSC in two ways. First, the poor quality and escalating cost of privately rented accommodation, combined with more restricted access to social housing, was putting additional pressure on deprived families. Problems included overcrowding, lack of space and amenities, rising rates of eviction, and longer waiting lists for social housing. Such stress factors were implicated in a higher risk of children experiencing abuse and neglect. Nonetheless, it was recognised that housing in the LA was relatively good compared to other parts of the country, particularly large cities. Second, new housing developments were thought to have led to a rise in the child population in the local area, with a proportion of those children requiring additional support. A particular concern for senior managers was that around 30,000 additional homes were planned in the local area over the next five years.

\section{Short term drivers}

Many participants considered that the local authority had put greater emphasis on partnership working in child protection over the past three years, which may have had a cumulative effect contributing to the recent spike. Specific measures included rolling out multi-agency training on specific issues such as child sexual exploitation, dissemination of risk assessment tools and protocols, and new organisational structures such as multi-agency safeguarding hub (MASH) and integrated Early Help (see below). These changes were thought to have led to greater awareness of risk indicators and safeguarding responsibilities among agencies, better information-sharing and more readiness to refer to CSC. There was widespread agreement that multi-agency working across Wokingham had improved in recent years, leading to better awareness amongst agencies of their respective roles and responsibilities for child safeguarding, and leading to more appropriate referrals. Co-location of staff from different agencies in MASH and Early Help had also helped to forge a common language and understanding around risk and decision-making. Social workers from 
CSC felt that other professionals from other agencies were now more able to recognise signs of abuse and neglect, more alert to issues such as child sexual exploitation, and more comfortable sharing information or asking for advice:

'I think if you are thinking about why CP has gone up. I think... multi-agency working. People are becoming more knowledgeable, training has probably got a lot better, you know people ring for advice, they know that they can and actually through those discussions sometimes they may be noticing something that they haven't noticed before...' [P8].

Over the past two years, due to increased demand for targeted preventative work, the LA had decided to bring together Early Help provision into an integrated service with close links to CSC and a focus on time-limited casework (usually six weeks). The main benefits were described as having one point of access for all early help services, a multi-agency approach, a common assessment process and case recording system. These changes, along with the introduction of Signs of Safety (see below) had given practitioners a clearer process for working with families around safeguarding concerns, setting and reviewing goals, and escalating to CSC if the situation did not change. While participants were generally positive about the new way of working, there was also recognition that demand for Early Help had gone up, as indicated by long waiting lists for allocation, and that higher expectations of change were sometimes hard to reconcile with a shorter intervention period. The bottleneck in provision was forcing Early Help services to find ways of managing demand, while making it harder for CSC to divert cases that normally would not require statutory intervention.

"We have had to become more focussed with our time, swifter about making decisions about things. Actually I think as managers we have to have more clarity about where cases are going so that we don't have drift.....so I think it forces us to review the work being done so we can make way." [P20]

"I think the biggest impact I've seen has been the Early help waiting list because obviously caseloads have gone up in duty which mean we have more going through to Early Help so they get really backed up and then we're having rereferrals because they are waiting for the intervention and it becomes a bit of a vicious circle really, particularly for adolescents". [P6].

Particular concerns were expressed about adolescents experiencing problems such as self-harm and sexual exploitation. Often these young people were having to wait six to eight weeks for targeted youth work interventions (offered as part of Early Help) during which time the risks to the child might escalate. Some participants also felt that short term targeted interventions were difficult to implement for young people, who were less inclined to engage with professionals and needed time 
to build trust and rapport with adults. Others felt that a targeted approach could be effective but needed to be supplemented by universal provision that could pick up children and young people when short-term interventions had finished.

Signs of Safety (SoS) had been introduced in the LA in 2014 and moved into a second phase of implementation in September 2017. Participants thought the approach had had a positive impact on practice, particularly in terms of developing more collaborative safety plans with families and their networks. SoS was also considered to have improved joint working across agencies and within social care, partly due to stakeholders being open to a more strengths-based approach to discussing and assessing risk to families. Some practitioners thought that the SoS approach brought greater structure and clarity to their work, e.g. by providing a clear 'bottom line' to parents, families and professionals about the steps necessary to establish safety for children, including clear actions and timescales for those actions. In cases of domestic violence, for example, it was suggested that prior to the introduction of SoS a common intervention might have been to send the perpetrator on a course and monitor the situation at home for improvement; now there was more emphasis on achieving concrete indicators of safety for the child within shorter and more specific timescales:

\begin{abstract}
'SoS is much more about building safety so it's much more instant.. You can see whether it's working... you can add to it but actually you know at a much earlier stage whether you have got sufficient safety or not. I think that sort of helps. I think there is a real focus on not letting things drift and I think that could have some impact on numbers as well.' [P20]
\end{abstract}

The SoS approach was therefore considered to have led to speedier, more assertive decisionmaking, and more focused information sharing between agencies. These factors were widely thought to have contributed to the rise in child protection work:

\begin{abstract}
I think it might have an impact with more child protection because of the joint training and more focussed discussions with families and working in the SoS way which for me is much more child friendly. I think it enables us to find more information out and use ways to explore in more detail and so I would say my team are much more skilled now at seeing danger, at identifying risks. So I think that possibly that has meant things have shifted up to child protection because we feel we have better evidence to be able to say we feel the children are at risk of significant harm here and 'this is why' you know. [P7]
\end{abstract}

While Ofsted inspections had been favourable on the whole, several participants mentioned the impact of a joint targeted area inspection (JTAI) in early 2017, which had focused on neglect. The inspection had raised awareness of neglect indicators in more affluent households, especially in 
relation to emotional neglect, as well as the cumulative impact of neglect on children's development. Increased awareness of neglect was thought by some practitioners to have led to more strategy discussions and child protection conferences in cases that might previously have been considered to be at a lower level of risk. It was also thought that recommendations from JTAI had contributed to a more assertive approach to long-running CIN cases where neglect was a factor:

'One thing that came out of it I think for our team was the cumulative impact of neglect. By the time someone reaches their teenage years...they have had years of this - so it might be what has always been considered low level but has always been in and out of Early help...CIN...Early help...CIN...CP...Early help...CIN...you know - through that pattern we have to get to a stage where we think "actually what's this done to the child now?"' [P2]

Practitioners thought that senior leadership in CSC had supported them to be more decisive in assessment and care planning, in order to address risks to children in a timely fashion and avoid drift. One manager thought that this constituted a change of culture in the LA, where previously there had been more emphasis on longer term work and keeping children with their families.

'I think there is more of an emphasis on keeping an eye on permanence and actually not letting those cases - maybe child in need cases you know - in a sense go along. So you know if we are not able to go along and create sufficient safety then we will escalate it and I think that's one of the themes for me."[P22].

Another senior manager echoed this perception. She thought that a decade ago agency leaders had fostered a culture of practice focused on keeping families together and this had caused certain cases - particularly in relation to neglect - to drift in CIN provision. Recently the LA had adopted a more interventionist culture, resulting in some children being admitted into care as adolescents when they had been known to services for several years.

\section{Discussion}

The principal long term drivers of demand identified in the study - increasing hardship and need in communities combined with the erosion of preventative services for children and young people largely reflect the 'poverty plus cuts' narrative described earlier. These factors were linked to broader social problems such as housing, employment, and crime. To take the example of housing overcrowded, unhealthy and poor quality homes have long been a defining characteristic of families involved in the child protection system (Bywaters et al., 2016; Thoburn, 2000). In England, such problems have been exacerbated by an acute shortage of supply, particularly of 'affordable' and 
social housing, putting poor families at greater risk of homelessness, eviction and exploitation by landlords (Dorling, 2014). Other indicators of deprivation, including unemployment, low income, debt, and the breakdown of support networks, have also been tied to broader structural changes, such as widening inequality (Wilkinson \& Pickett, 2010), the growing precarity of social arrangements (Standing, 2011), and the increasing power of global private capital (Meek, 2014). Acting cumulatively and over a period of time, these factors contribute to a rising tide of poverty and exclusion, which can be expected to affect an increasing proportion of families in any area. This context may partly account for Homeshire's concern about the impact of new housing developments, or indeed any growth in the child population - these children would be increasingly likely to need social care provision.

In addition to long term drivers, the study pointed to other factors seen as driving medium to short term fluctuations in demand. In Homeshire these included leadership priorities, the impact of inspections, new practice models, the resourcing of Early Help, and partnership arrangements. They reflect alternative explanations to the poverty-plus-cuts narrative, with more emphasis on the role of agencies and professionals in shaping provision, determining eligibility, and administering thresholds for statutory intervention. One question this raises is how long- and short-term drivers of demand will interact, given that agencies are likely to respond to increased pressure on services, e.g. by managing demand and rationing resources. In Homeshire, short term factors were thought to have converged on a more robust response to abuse and neglect, contributing to the spike in referrals and CP activity. Reasons for this ranged from accountability pressures to improvements in practice, and the 'bottom line' represented by the statutory duty to protect children. From the LA's perspective, it could be argued that a shift to late intervention was a reasonable response given the socio-political environment and the institutional and economic constraints in which it was operating. Given the resource implications, a key question is how to predict the future direction of demand when a combination of short and long-term drivers are in play. Should a spike in referrals or CP plans be seen as a step change in demand or as a symptom of volatility in a system under strain? While the latter may seem preferable (CP plans might go down again next year) unpredictable variation in demand may be bad news for services already operating at or near full capacity. A study of accident and emergency departments by Lane et al. (2000) showed that a system with little or no slack (in terms of resource utilisation) would not easily cope with a pulse in demand and could collapse entirely (Lane et al., 2000). 
It is interesting to consider the role of Early Help in this picture of demand. The term was introduced in the Munro Review as an alternative to 'early intervention', which had become associated with a rather narrow and programmatic approach to service delivery (Munro, 2011). The idea of EH was to work in partnership with families who were starting to experience difficulties, bridging the gap between community-based family support and early intervention (Frost et al., 2015). The policy focus on infancy and early childhood sometimes associated with the early intervention agenda (e.g. Allen, 2011), was given less prominence in $\mathrm{EH}$, the emphasis being on early prevention of problems regardless of the child's age (as reflected in the concern with adolescents in the findings). However, like early intervention, EH was conceived as a specialist service, aimed at children and families with additional needs referred from the community. Although non-statutory, this type of 'prevention' is to be distinguished from services such as youth clubs and Sure Start centres, or the kind of support provided by voluntary sector organisations. Indeed, the picture of EH that emerged from this study suggest more than anything an additional layer of CSC sitting just below the threshold for statutory services and organised in a similar way, i.e. on the basis of thresholds, assessments, and time-limited casework. A key rationale for this strategy is that allocating resources to targeted prevention might soften the impact of cuts to services in the community, the idea being that even if there was an increase in children with additional needs and problems, they could be identified and supported before they needed statutory services. However, there was little evidence to support this in Homeshire, where the development of targeted EH had not prevented a surge in demand for child protection. North Yorkshire and Essex, two LAs rated 'outstanding' by Ofsted in 2018, both reported increases in CP plans in the latest government figures (DfE, 2018) despite having effective and wellresourced EH services. Of course, the financial pressure on LAs means that the money available for EH may simply not be enough. A moot point is whether funding targeted intervention is necessarily a better use of scarce resources than funding community-based provision. Here the logic of late intervention is self-fulfilling - the more complex or high risk the situation the easier it is to demonstrate effectiveness, i.e. a problem solved and potential crisis averted. From a statutory perspective, EH is always going to 'look' more effective than a youth club because it is easier to identify individuals who may have diverted from more intensive (and expensive) forms of provision. But this is only a partial view of effectiveness; the contribution of universal services cannot be measured in the same way because they serve the needs of a community - their knock-on effects are dispersed among a range of providers including health services and the criminal justice system.

As noted earlier, one of the key challenges for CSC lies in balancing the regulatory element of demand (protecting children from abuse) with the broader welfare element (supporting families and 
communities so that children can thrive). In the 1989 Children Act, the enabling legislation for CSC in England, this balance is formalised in the distinction between services provided under Section 17 (children in need) and Section 47 (child protection). LAs have statutory duties under both but the shift to late intervention may be leading to an erosion of Section 17 provision, with family support becoming the remit of EH and 'everything else' escalating to CP. Given rising demand and falling budgets this is an understandable direction for LAs to take - but, it could be argued, runs contrary to the spirit of the legislation. Moreover, as Sparrow (2000) points out, effective regulation often comes at the cost of citizen-shaped services - the former involves a modus operandi quite different from the latter, as families who have been subject to Section 47 inquiries would no doubt attest. In most regulatory environments there are interactions between different elements of the system. Unfortunately, a CSC system oriented around policing families seems likely to produce many more families who need policing.

\section{Conclusion}

This paper has reported on a particular configuration of long- and short-term drivers of $\mathrm{CP}$ in a single LA. Its findings are not generalizable although there may be elements of transferability to other areas, particularly in relation to long-term demand drivers that reflect national trends as well as local conditions. Other limitations of the study include its cross-sectional nature, with data collected at a point in time when sensitivities to demand pressures were particularly heightened, and a purposive rather than representative sample of participants. Its main strength lies in the contextual detail provided by the qualitative data, which addresses the interaction of different demand drivers. In summary, demand in Homeshire increased as a result of greater normative need ('poverty plus cuts') combining with operational changes, e.g. to leadership, multi-agency working, and decision-making, to increase the number of children identified as needing statutory intervention. More targeted use of Early Help had so far not obviously reduced the need for services. One implication beyond this particular LA is that the 'poverty-plus-cuts' drivers will continue to gradually push up rates of CP and encourage the shift to late intervention. Short-term factors are likely to differ across local areas and regions, both in their nature and their effect, so that fluctuations from year-to-year may accentuate or counteract this pattern. However, given their acute financial pressures, LAs will have less capacity to absorb short-term pulses in demand. More broadly, national policymakers seem to have underestimated the risk that local government funding cuts would incur higher levels of spending in parts of the system where statutory duties apply. Currently we have a very limited understanding of these interactions, which necessarily constrains the search for workable solutions. 


\section{References}

Action for Children, National Children's Bureau \& The Children's Society (2017) Turning the Tide. available online: https://www.childrenssociety.org.uk/what-we-do/resources-andpublications/turning-the-tide

Association of Directors of Children's Services (ADCS). (2018) Research Report: Safeguarding Pressures Phase 6. Association of Directors of Children's Services, Manchester.

Bradshaw, J. (1972) Taxonomy of social need'. In: Problems and progress in medical care : essays on current research (ed G. McLachlan), pp. 71-82. Oxford University Press, Oxford.

Braun, V., Clarke, V., Hayfield, N. \& Terry, G. (2019) Thematic analysis. In: Handbook of Research Methods in Health Social Sciences (ed P. Liamputtong), pp. 843-860. Springer, Singapore.

Butler, P. (2018) Tory-run Northamptonshire county council bailed out by government. available online: https://www.theguardian.com/society/2018/nov/29/tory-run-northamptonshire-countycouncil-bailed-out-by-government

Bywaters, P. (2017) Identifying and Understanding Inequalities in Child Welfare Intervention Rates: comparative studies in four UK countries. Briefing Paper 2: UK Four Country Quantitative Comparison. available

Bywaters, P., Bunting, L., Davidson, G., Hanratty, J., Mason, W., McCartan, C. \& Steils, N. (2016) The relationship between poverty, child abuse and neglect: an evidence review. Joseph Rowntree Foundation, York.

Department of Health. (1995) Messages from Research. HMSO, London.

Dorling, D. (2014) All that is solid: How the great housing disaster defines our times, and what we can do about it. Penguin, London.

Featherstone, B., Gupta, A., Morris, K. \& White, S. (2018) Protecting children: A social model. Policy Press, Bristol.

Frost, N., Abbott, S. \& Race, T. (2015) Family support: Prevention, early intervention and early help. John Wiley, London.

Hood, R. (2019) What to measure in child protection? The British Journal of Social Work, 49, 466484.

Hood, R., Goldacre, A., Gorin, S. \& Bywaters, P. (2019) Screen, ration and churn: Demand management and the crisis in children's social care. In: The British Journal of Social Work. Hood, R., Grant, R., Jones, R. \& Goldacre, A. (2016) A study of performance indicators and Ofsted ratings in English child protection services. Children and Youth Services Review, 67, 50-56. Howe, D. (2014) The compleat social worker. Palgrave Macmillan, Basingstoke.

Lane, D.C., Monefeldt, C. \& Rosenhead, J.V. (2000) Looking in the wrong place for healthcare improvements: A system dynamics study of an accident and emergency department. Journal of the Operational Research Society, 51, 518-531.

Lewis, M.A. \& Widerquist, K. (2001) Economics for Social Workers: The Application of Economic Theory to Social Policy and the Human Services. Columbia University Press, New York.

Manning, A. (2003) Monopsony in motion: Imperfect competition in labor markets. Princeton University Press.

Maslow, A. (1970) Motivation and personality, 2nd Edition. Harper \& Row, New York. Meek, J. (2014) Private island: Why Britain now belongs to someone else. Verso, London.

Munro, E. (2011) The Munro Review of Child Protection: Final Report. A child-centred system. TSO, London.

National Audit Office. (2019) Pressures on Children's Social Care. NAO, London.

O'Hara, M. (2015) Austerity bites: A journey to the sharp end of cuts in the UK. Policy Press, Bristol. Ofsted (2015) Joining the dots... Effective leadership of children's services. available online: www.gov.uk/government/organisations/ofsted 
Sparrow, M.K. (2000) The regulatory craft: controlling risks, solving problems, and managing compliance. Brookings Institution Press, Washington, DC.

Standing, G. (2011) The Precariat: the new dangerous class. Bloomsbury, London.

Thoburn, J., Wilding, J. \& Watson, J. (2000) Family Support in Cases of Emotional Maltreatment and Neglect. The Stationery Office, London.

Turnell, A. (2017) The Signs of Safety Comprehensive Briefing Paper (4th Edition). available online:

http://sofs.s3.amazonaws.com/downloads/Briefing\%20Paper\%20v2-1.pdf

Wijedasa, D., Warner, N. \& Scourfeld, J. (2018) Exploratory analyses of the rates of children looked after in English local authorities (2012-2017). What Works Centre for Children's Social Care, London. Wilkinson, R. \& Pickett, K. (2010) The spirit level: why equality is better for everyone. 